\title{
Radiation dose-fractionation effects in spinal cord: comparison of animal and human data
}

\author{
Jian-Yue Jin ${ }^{1}$ - Yimei Huang ${ }^{2} \cdot$ Stephen L. Brown ${ }^{2}$. \\ Benjamin Movsas ${ }^{2} \cdot$ Joseph Kaminski $^{1} \cdot$ Indrin J. Chetty $^{2}$ • \\ Samuel Ryu ${ }^{3} \cdot$ Feng-Ming (Spring) Kong ${ }^{1}$
}

Received: 14 April 2015 / Accepted: 26 July 2015 / Published online: 14 August 2015

(C) The Author(s) 2015. This article is published with open access at Springerlink.com

\begin{abstract}
Purpose Recognizing spinal cord dose limits in various fractionations is essential to ensure adequate dose for tumor control while minimizing the chance of radiationinduced myelopathy (RIM). This study aimed to determine the $\alpha / \beta$ ratio of the spinal cord and the cord dose limit in terms of BED50, the biological equivalent dose (BED) that induces $50 \%$ chance of RIM, by fitting data collected from published animal and patient studies.

Methods RIM data from five rat studies; three large animal studies on monkeys, dogs, and pigs; and 18 patient studies were included for the investigation. The $\alpha / \beta$ ratios were derived, respectively, for rat (group A), large animal (group B), patient (group C), and combined data (group D).

Results The $\alpha / \beta$ ratio (and its $95 \%$ confidental interval) was $4.1(3.2,5.0)$ or $3.6(2.6,4.6)$ Gy for group A, depending on fitting algorithms. It was $3.9(3.0,4.8), 3.7(2.2,8.2)$ and $3.9(3.0,4.9)$ for groups $\mathrm{B}, \mathrm{C}$, and $\mathrm{D}$, respectively. BED50 was 111 Gy for the combined data. It corresponds to a D50 of $73.4 \mathrm{~Gy}$ in $2 \mathrm{~Gy} / \mathrm{FX}$, or $19.0 \mathrm{~Gy}$ in single fraction. BED5, which is the BED to induce $5 \%$ of RIM, was calculated to be $83.9 \mathrm{~Gy}$. It corresponds to D5 of $55.4 \mathrm{~Gy}$ in $2 \mathrm{~Gy} /$ FX, or 16.2 Gy in single fraction.
\end{abstract}

Jian-Yue Jin

jjin@gru.edu

1 Department of Radiation Oncology, Georgia Regents University, Augusta, GA 30912, USA

2 Department of Radiation Oncology, Henry Ford Hospital, Detroit, MI 48202, USA

3 Department of Radiation Oncology, Stony Brook University, Stony Brook, NY 11794, USA
Conclusion The study showed that all four groups had similar $\alpha / \beta$ ratios close to $3.9 \mathrm{~Gy}$, suggesting that the spinal cord has a similar fractionation effect for different species, including human beings.

Keywords Cord dose tolerance $\cdot \alpha / \beta$ ratio $\cdot$ Myelopathy . Dose fractionation effect

\section{Introduction}

Radiation-induced myelopathy (RIM) is one of the most severe complications for radiation treatment of cancers in the neck, thoracic, and abdominal regions. The human spinal cord was estimated to have a probability of RIM of less than $5 \%$ in 5 years following a dose of 47-50 Gy delivered in a standard $1.8-2 \mathrm{~Gy} /$ fraction schedule $[1,2]$. The spine is also one of the most frequent sites for metastases. The metastatic tumor often invades into the spinal cord, compresses the cord, and causes an emergency, which requires radiation treatment and surgical intervention. A conventional radiation regimen of $30 \mathrm{~Gy}$ in 10 fractions is often used to manage patients with cord compression, preceded or followed by a decompression surgery. Recently, a radiosurgical decompression approach has been proposed to manage these patients [3]. In addition, linear accelerator-based stereotactic radiosurgery (SRS) and stereotactic body radiotherapy (SBRT) have been frequently used to treat spine metastasis without cord compression [3-5]. A RIM model that reflects the fractionation effect of the spinal cord is needed to optimize the radiation dose and fractionation scheme.

The fractionation effect, represented by the $\alpha / \beta$ ratio in the linear quadratic model, has been well studied for the spinal cord on rats [6-10]. These prospective studies showed that the $\alpha / \beta$ ratio of the rat spinal cord ranged from 1.8 4.6 Gy, depending on the site (cervical or lumbar spine) and the study 
[11]. However, applying rat models to humans is intrinsically complicated because their anatomy and lifespan are quite different from humans. The rat data may also have relatively large experimental uncertainties due to very narrow radiation fields used to spare the normal structures. The narrow radiation field usually has a non-flat dose profile across the field. Thus, even a normal experimental setup uncertainty may induce a relatively large dosimetric uncertainty.

Studies have been carried out on larger animals, such as dogs, monkeys, and pigs, for the RIM in several different fractionation schemes 12. However, large animal studies are expensive. The data from each of these experiments were not sufficient to derive the $\alpha / \beta$ ratio. Many retrospective studies have reported RIM incidences in humans at various cord doses and fractionations. An $\alpha / \beta$ ratio of $0.87 \mathrm{~Gy}$, with a $95 \%$ confidence interval $(\mathrm{CI})$ of $(0.54,1.19)$ Gy was derived from these limited data for the human cervical spine [12]. However, human data are usually highly limited in power, because of the rare occurrence and nature of retrospective documentation. These data are often anecdotal, have limited number of data points, small range of doses, inconsistent endpoints, and sometimes, inaccurate cord dose calculations. For these reasons, the $\alpha / \beta$ ratio derived from these data may be unreliable.

The aims of this study were to compare the $\alpha / \beta$ ratios of the spinal cord between patients and animals by re-analyzing existing data in the literature, and to develop a RIM model from the combined data. Because the anatomy and life span between species is different, the animal data were further separated into small animal (rat) and large animal groups for comparison. Several unique approaches were used in the reanalysis: (1) normalization and non-linear regression were used to pool all rat data together to increase the statistic power and reduce the effect of large dosimetric uncertainties; (2) data of large animal were grouped together to derive an $\alpha / \beta$ ratio for this group; and (3) Monte Carlo simulation was used to correct a potential dosimetric error for one patient study, which provided the key data for deriving the $\alpha / \beta$ ratio on patients.

\section{Material and methods}

\section{Data collection and correction}

All available animal and patient studies were collected from literature searches using key words of "radiation induced myelopathy,", "radiation, myelopathy," "radiation, spinal cord complication," or "radiation, spinal cord injury." The data were separated into four groups: (A) small animals, (B) large animals, (C) patients, and (D) patients and large animals combined. To avoid the confounding effect of dose rate and volume effects, studies (or data points) with low dose rate
$(<0.1 \mathrm{~Gy} / \mathrm{min})$ or small volume ( $<2$ vertebral sections) were excluded. Because of the concern of large dosimetric uncertainties in small animal studies (rats and mice), small animal data were not included in the combined patient and animal group, and only those rat studies specifically designed for studying the fractionation effect were included in the small animal group. A total of 5 rat [6-10], 3 large animal [13-15], and 18 patient studies [16-31] were finally included in this investigation.

The data of five rat studies [6-10] in group A were shown in Table 1. They had six data sets, with the corresponding data points expressed as $(\mathrm{D} 50, n)$, where D50 is the total dose with $50 \%$ of RIM incidence, and $n$ is the fraction number. The data sets were further separated into the cervical (C) and thoracicolumber $(\mathrm{T} / \mathrm{L})$ spine subgroups. We noted that even within the same subgroup, there were large variations between different data sets. Two sources of experimental errors might contribute to these variations: (1) dose calibration uncertainty in the small animal experiments and (2) uncorrected quality factor between kilovolt and megavolt photons. The quality factor could be up to 1.12 for $50 \mathrm{kV}$ photons [32].

Normalization was performed for these data to reduce the variations among different studies so they could be analyzed together. This was based on the assumption that all the rats had similar radiation response after a single fraction of irradiation, and the variation among different studies was due to experimental uncertainties (dose calibration and the uncorrected quality factor). The average D50 value of all data sets at single fraction was first calculated (it was 22.5 Gy). The normalization was accomplished by multiplying a factor for each data set so that the D50 values at single fraction were all equal to the average value.

Group B included three animal studies on dogs, monkeys, and pigs [13-15]. Several data points in the dog and monkey studies were not included in the analysis because the irradiated cord length could have been less than two vertebral sections. As shown in Table 2, the data were presented as the percentage of RIM (PRIM) incidence, and the irradiated dose per fraction $(d)$ multiplying the number of fractions (n), or $d \times n$.

The patient data in group $\mathrm{C}$ had a total of 25 data points (RIM data at 25 different fractionation regimens). The data were also presented as (PRIM, $d \times n$ ) in Table 2. Among them, 16 data points were from 16 patient studies [16-31] previously compiled and analyzed for the $\alpha / \beta$ ratio $[12,35]$. These data were also reviewed in a Quantitative Analysis of Normal Tissue Effects in the Clinic (QUANTEC) paper [2]. Several of these studies had more than one data points, and others had the same dose-fractionation regimen, which were combined into a single data point. For the 16 data points, 7 were on C-spine, and 9 on thoracic (T)-spine. The other 9 data points were new data from a set of patient data collected in four hospitals in Netherlands [34]. That study reported that 19 of 43 patients 
Table 1 Data points of six data sets in five studies on rats specifically designed for the fractionation effect

\begin{tabular}{lllll}
\hline Study & $\begin{array}{l}\text { Radiation } \\
\text { fields }\end{array}$ & $\begin{array}{l}\text { Follow-up } \\
\text { time (days) }\end{array}$ & $\begin{array}{l}\text { Number of } \\
\text { data points }\end{array}$ & Data points (D50 [Gy], fraction [n]) \\
\hline Karger (C) [6] & PA & 300 & 4 & $(24.5,1) ;(34.3,2) ;(57,6) ;(88.6,18)$ \\
Ang (C2-T2) [7] & PA & 210 & 4 & $(22.4,1) ;(30.4,2) ;(43.4,4) ;(63,10)$ \\
Van der Kogel (C) [8] & Lateral & $\sim 365$ & 5 & $(19,1) ;(27,2) ;(37.8,5) ;(55,10) ;(80,30)$ \\
White (L) [9] & Lateral & 365 & 6 & $(24,1) ;(33,2) ;(46,4) ;(59,8) ;(68,15) ;$ \\
& & & & $(92,30)$ \\
Masuda (T) [10] & Lateral & 455 & 3 & $(25.5,1) ;(34.5,2) ;(46.6,4)$ \\
Van der Kogel (L) [8] & Lateral & $\sim 365$ & 7 & $(19.5,1) ;(27,2) ;(32.1,3) ;(36.8,5) ;$ \\
& & & & $(47.7,10) ;(60.4,15) ;(67.1,20)$ \\
\hline
\end{tabular}

$C$ cervical, $T$ thoracic, $L$ lumber developed myelopathy in the $\mathrm{T}$ spinal cord with the radiation dose ranging from 43 to $74 \mathrm{~Gy}$, and fraction size ranging from 1.9 to $3.6 \mathrm{~Gy} /$ fraction. The 43 patients were divided into nine groups according to the doses and fractionations. Patient data from spinal SRS or SBRT [2] were not included in this study due to the patient setup-related dosimetric uncertainty and the partial cord volume irradiation.
All the patient data were thoroughly evaluated with the original sources. Of note, three data points were modified as a result. Data point 1 was from a report of myelopathy after radiotherapy of breast cancers [19]. A $22.5 \mathrm{MeV}$ anteriorposterior electron beam was given to the chest wall and supraclavicular area. The prescription dose was 25 Gy in 2 fractions. The cord dose was recorded as $19 \mathrm{~Gy}$ in 2 fractions.
Table 2 Data points from 3 studies on large animals and 17 studies on patients for the radiation-induced myelopathy model

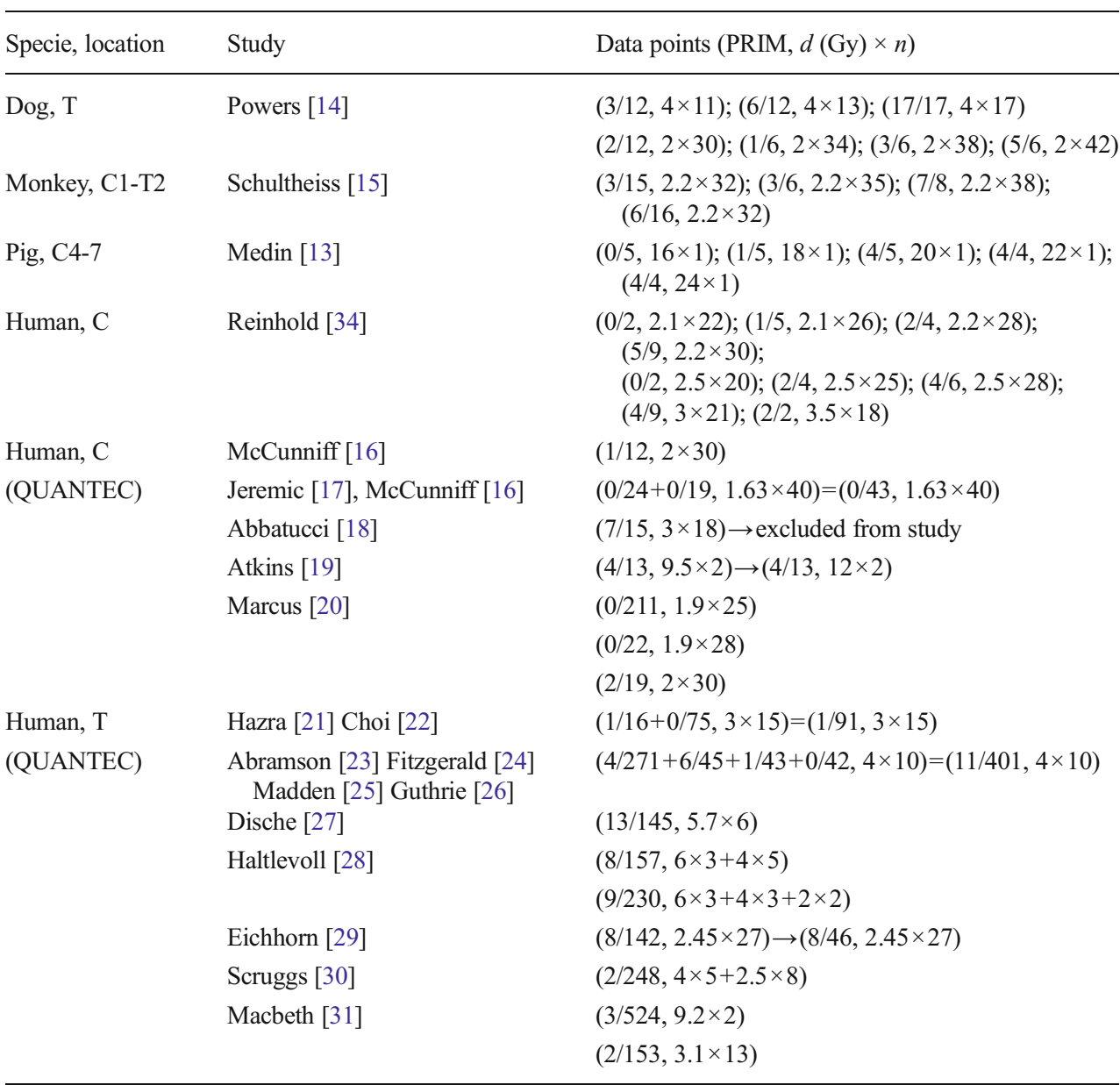

PRIM percentage of radiation-induced myelopathy=number of RIM/total number; $d$ dose/fraction, $n$ number of fractions, $C$ cervical, $T$ thoracic 
However, a Monte Carlo calculation suggested that the cord dose could be much higher. Figure 1 shows the dose distribution with Monte Carlo calculation of a $20-\mathrm{MeV}$ electron beam in AP direction delivered to the same region for a female patient. We noted that the maximum cord dose was as high as the prescription dose. Therefore, the cord dose for data point 1 was estimated to be 24 Gy in 2 fractions.

Data point 2 was from Eichhorn et al. [29]. The incidence of RIM was first listed as $8 / 46$ [35], but might be subsequently misquoted as $8 / 142$ in later papers $[2,12]$. The correct value, which is $8 / 46$, was used in our study. Data point 3 was from a French study [18]. Incidence of RIM was first listed as $8 / 20$ $[35]$ and then $7 / 15$ in later papers $[2,12]$ for a cord dose of $3-G y \times 18$. However, there were 94 patients who received a $3 \mathrm{~Gy} \times 18$ prescription dose in the control group [18]. Because the cord dose of these 94 patients was not clearly provided, the incidence of RIM for this data point was difficult to verify and the data point was excluded from the final analysis.

\section{Data analysis}

For group A, a conventional linear regression method [11] was first used to derive the $\alpha / \beta$ ratio as well as the biological equivalent dose (BED) at $50 \%$ of RIM probability (BED50). The linear equation was derived directly from a BED equation,

$\mathrm{BED} 50=\mathrm{D} 50 \cdot\left[1+\frac{\mathrm{D} 50}{n \cdot(a / \beta)}\right]$
Dividing both sides of Eq. (1) to a term of D50 BED50, we have,

$\frac{1}{\mathrm{D} 50}=\frac{1}{\operatorname{BED} 50}+\frac{1}{\operatorname{BED} 50 \cdot(a / \beta)} \cdot \frac{\mathrm{D} 50}{n}$

Equation (2) is a linear function of $(1 / \mathrm{D} 50, \mathrm{D} 50 / n)$. The data set of $(\mathrm{D} 50, n)$ was converted into $(1 / \mathrm{D} 50, \mathrm{D} 50 / n)$ for linear regression analysis. The values of BED50 and $\alpha / \beta$ were derived by best fitting the data into the linear equation. Such analysis was performed for each data set for the original data, and the combined data set for each subgroup after normalization.

The linear regression approach had to convert the data point $(\mathrm{D} 50, n)$ into $(1 / \mathrm{D} 50, \mathrm{D} 50 / n)$, and thus propagate the large uncertainty in D50 into both variables. To reduce the effect of such data conversion, a non-linear regression approach was also developed to derive the values of BED50 and $\alpha / \beta$. The data set of $(\mathrm{D} 50, n)$ was directly fitted into a non-linear equation,

$\mathrm{D} 50=\left\{\left[(\alpha / \beta)^{2} \cdot n^{2}-4 n \cdot \alpha / \beta\right]^{1 / 2}-n \cdot \operatorname{BED} 50 \cdot \alpha / \beta\right\} / 2$

which is the direct solution of Eq. (1).

For groups B, C, and D, the following logistical RIM model was used to fit the data:

$$
\begin{aligned}
\operatorname{PRIM}(d, n) & =1 /\left[1+(\mathrm{BED} 50 / \mathrm{BED})^{k}\right] \\
& =1 /\left[1+\left(\frac{\mathrm{BED} 50}{d \cdot n \cdot(1+d / \alpha / \beta)}\right)^{k}\right],
\end{aligned}
$$

Fig. 1 Monte Carlo calculated dose distribution of an $\mathrm{AP}$ $20 \mathrm{MeV}$ electron beam to a female patient

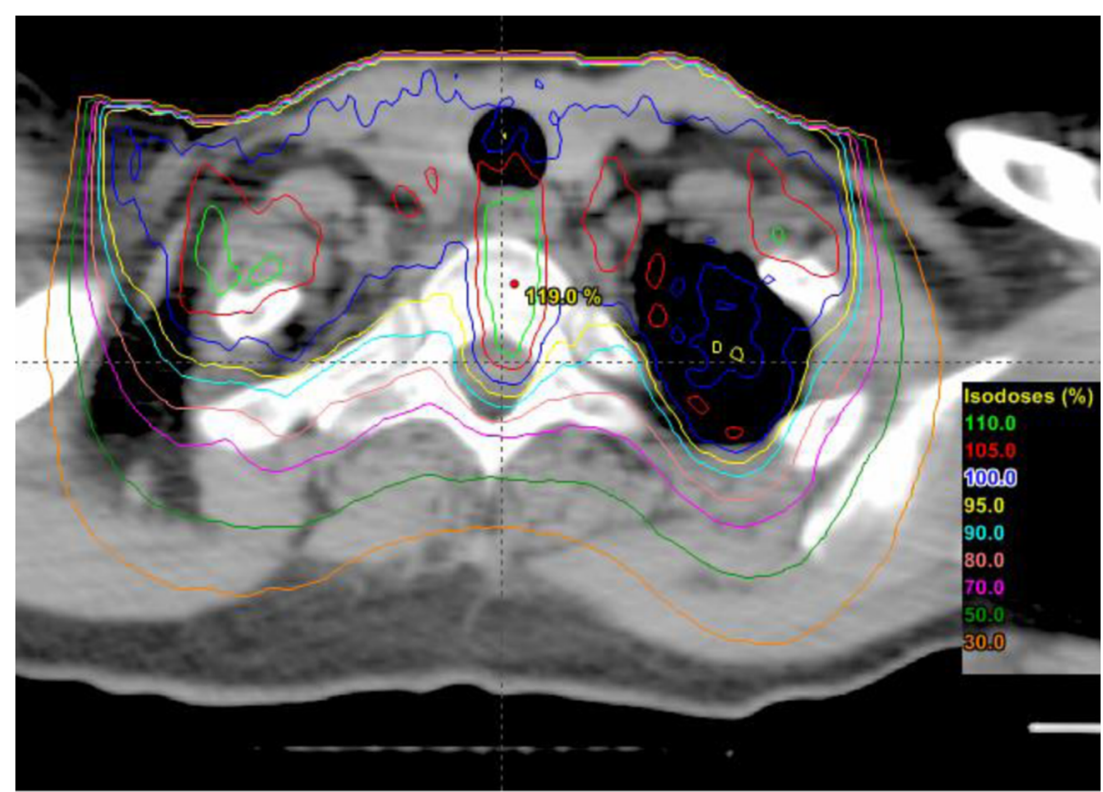


It should be noted that for several patient data points, multiple fractionation regimens were used in a treatment course. The sum of BED in each fractionation regimen was used for Eq. (4) in such situations. The sum of BED was expressed as

$\mathrm{BED}=\sum_{i=1}^{m} d_{i} \cdot n_{i} \cdot\left(1+d_{i} / \alpha / \beta\right)$

where $d_{i}$ and $n_{i}$ are the dose and fraction number of the $i$ th regimen, and $m$ is total number of regimens. Three parameters, $\alpha / \beta$, BED50, $k$, and their corresponding $95 \%$ CI were thus determined from the fitting program.

\section{Results}

\section{Fractionation effect from the rat studies}

Figure 2a, b show the original six data sets of the rat studies (group A) plotted as (1/D50 vs D50/n) for linear regression, and (D50 vs $n$ ) for non-linear regression, respectively. Figure 2c, d show the same data after normalization, as well as their linear and non-linear regressions, respectively. The large inter-study variations in Fig. 2a, b were dramatically reduced after normalization. The data were clearly separated into $\mathrm{C}$-spine and T/L-spine subgroups, and the inter-study variation within each subgroup was comparable to the intrastudy variation.

Table 3 shows the values of $\alpha / \beta$ ratio and BED50 after regression analyses. Despite large inter-study variation in the original data, the $\alpha / \beta$ ratios were relatively consistent within each subgroup. The average $\alpha / \beta$ ratios were 2.4 and 4.8 Gy for the $\mathrm{C}$-spine and $\mathrm{T} / \mathrm{L}$-spine subgroups, respectively, before normalization. They were slightly reduced to 2.2 and 4.1 Gy after normalization. The overall $\alpha / \beta$ ratios and their $95 \% \mathrm{CI}$ derived from the combined data sets were $2.3(1.5,3.0) \mathrm{Gy}$ and $4.1(3.2,5.0) \mathrm{Gy}$, respectively, for the $\mathrm{C}$ - and T/L-spine subgroups, using the linear regression. They became $2.6(2.1,3.2)$ Gy and $3.6(2.6,4.6)$ Gy, respectively, using the non-linear regression. In summary, the $\alpha / \beta$ ratios were slightly changed by the normalization and the different regression approaches, and consequently, the difference in $\alpha / \beta$ ratios between the two subgroups (C- and T/L-spines) was reduced, especially using the non-linear regression. As to be discussed, the smaller $\alpha / \beta$ ratio in the $\mathrm{C}$-spine subgroup could be due to shorter follow-up time. Thus, the values of the T/L-spine subgroup would be used for comparison to the large animal and patient data.
Fig. 2 Re-analysis of the $\alpha / \beta$ ratio for the six data sets from five rat studies specifically designed for studying fractionation effect using linear and non-linear regression. a Original data plotted $(1 / \mathrm{D} 50, \mathrm{D} 50 / n)$ for linear regression. b Original data plotted (D50, $n$ ) for non-linear regression. c Normalized data plotted (1/D50, $\mathrm{D} 50 / n)$ and linear regression of the combined data for $\mathrm{C}$ - and $\mathrm{T} / \mathrm{L}$ spine subgroups. d Normalized data plotted (D50, $n$ ) and nonlinear regression of combined data sets for the two subgroups
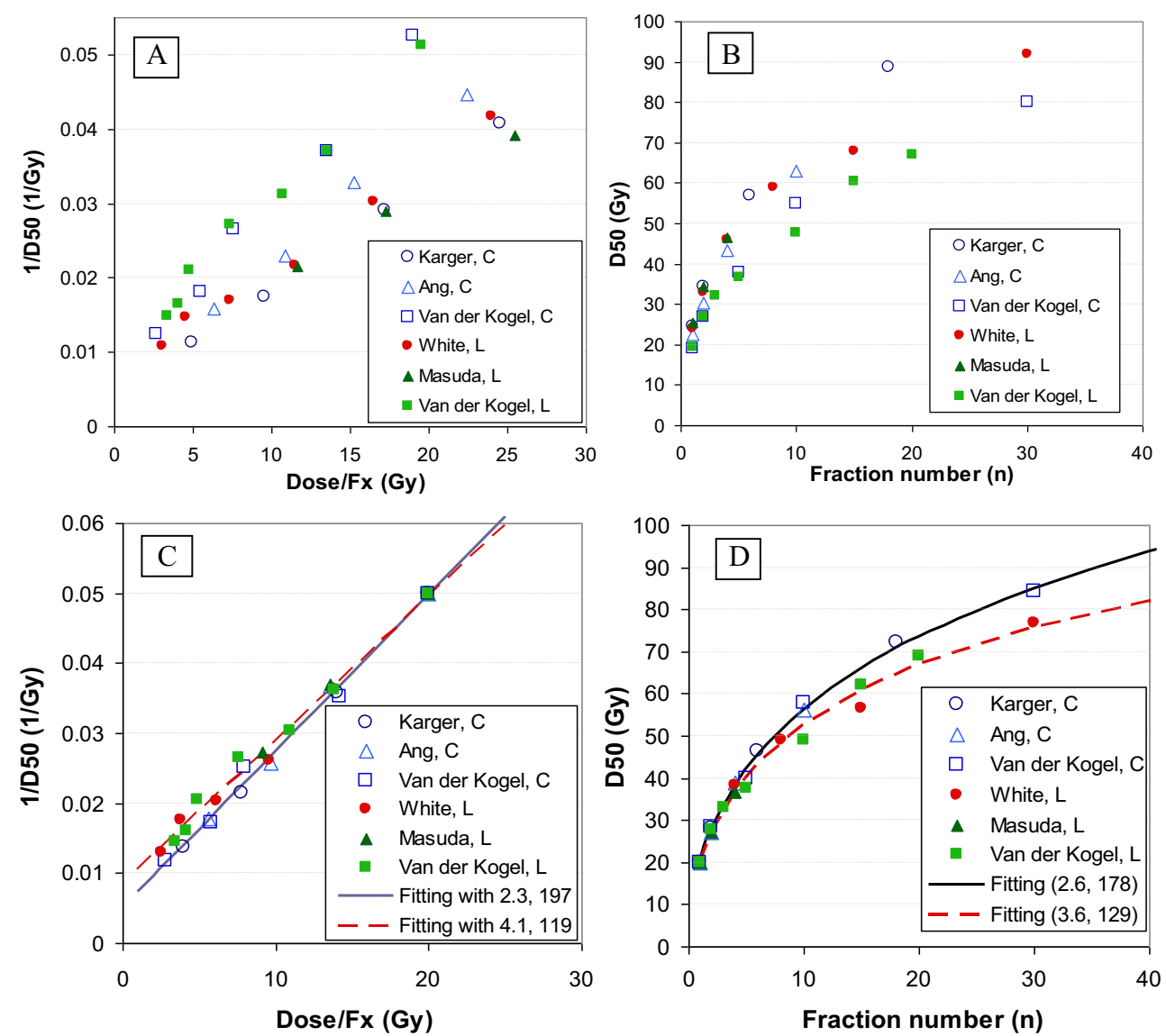
Table 3 Values of $\alpha / \beta$ ratio and BED50 from six data sets in five studies with and without normalization. The data were divided into cervical (C)-spine and thoracicolumber $(\mathrm{T} / \mathrm{L})$ spine groups

\begin{tabular}{|c|c|c|c|c|c|c|}
\hline \multirow[t]{2}{*}{ Subgroup } & \multirow[t]{2}{*}{ Study } & \multirow[t]{2}{*}{ Regression } & \multicolumn{2}{|l|}{ Original } & \multicolumn{2}{|c|}{ After normalization } \\
\hline & & & $\alpha / \beta$ (Gy) & BED50 (Gy) & $\alpha / \beta$ (Gy) & BED50 (Gy) \\
\hline \multirow[t]{6}{*}{ C-Spine } & Karger [6] & Linear & 2.3 & 286 & 1.9 & 234 \\
\hline & Ang [7] & Linear & 2.3 & 238 & 2.1 & 211 \\
\hline & Van der Kogel [8] & Linear & 2.5 & 167 & 2.6 & 174 \\
\hline & Average & Linear & 2.4 & 230 & 2.2 & 207 \\
\hline & Overall & Linear & $N A$ & $N A$ & $2.3(1.5,3.0)$ & $198(142,235)$ \\
\hline & Overall & Non-linear & $N A$ & $N A$ & $2.6(2.1,3.2)$ & $178(153,203)$ \\
\hline \multirow[t]{6}{*}{ T/L-Spine } & White [9] & Linear & 4.8 & 149 & 3.9 & 124 \\
\hline & Masuda [10] & Linear & 5.2 & 149 & 4.1 & 117 \\
\hline & Van der Kogel [8] & Linear & 4.3 & 111 & 4.3 & 114 \\
\hline & Average & Linear & 4.8 & 136 & 4.1 & 118 \\
\hline & Overall & Linear & $N A$ & $N A$ & $4.1(3.2,5.0)$ & $119(100,138)$ \\
\hline & Overall & Non-linear & $N A$ & $N A$ & $3.6(2.6,4.6)$ & $129(109,150)$ \\
\hline
\end{tabular}

\section{Fractionation effect from large animal and human data}

Figure 3a , b show the plots of the large animal (group B) and patient data (group C) with the best fitting of the RIM model. The original data points 1,2, and 3 before correction were also

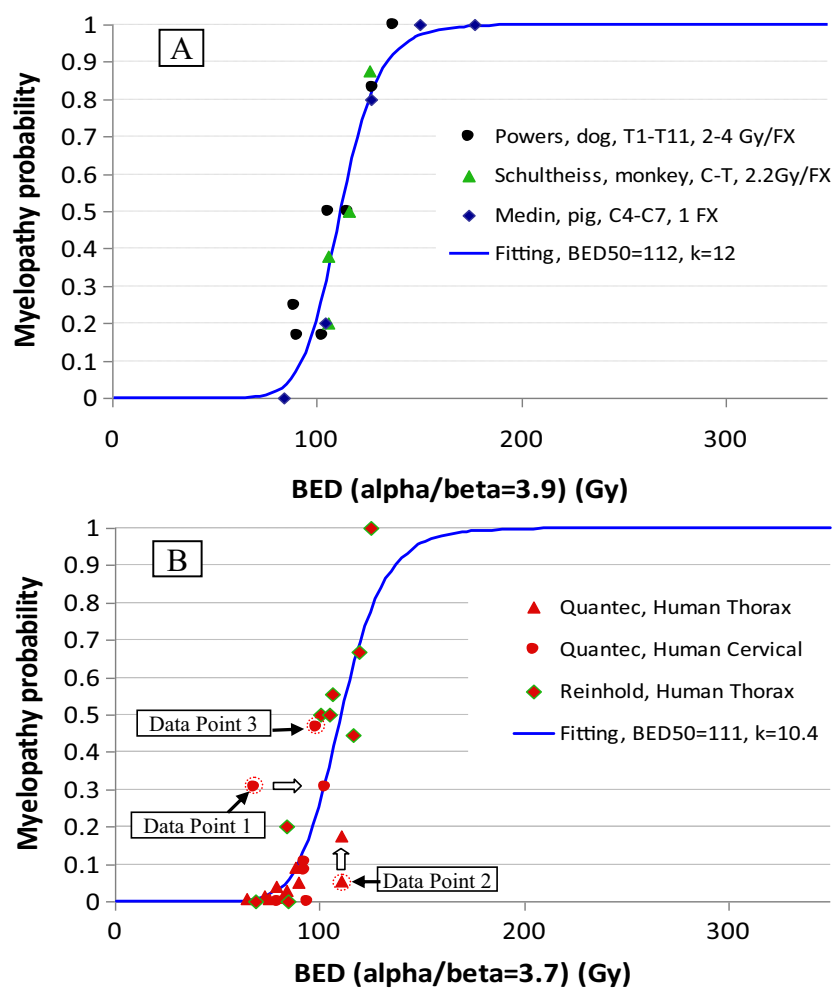

Fig. 3 Fitting the data with a logistic radiation-induced myelopathy (RIM) model. The data are plotted as percentage of RIM versus BED. BED was calculated from dose/fraction and number of fractions using the best fitting $\alpha / \beta$ ratio. a Large animal data. b Patient data. Data points 1, 2, and 3 before correction were also plotted for comparison shown in Fig. $3 \mathrm{~b}$ for comparison. The best fitting parameters and their $95 \%$ CI were $\alpha / \beta=3.9(3.0,4.8)$ Gy, BED50 $=112$ $(101,128) \mathrm{Gy}$, and $k=12(7.0,21)$ for group $\mathrm{B}$; and $\alpha / \beta=3.7$ $(2.2,8.2) \mathrm{Gy}, \mathrm{BED} 50=111(86,148) \mathrm{Gy}$, and $k=10.4(5.8$, 19.2) for group C. These data were also listed in Table 4 for comparison between the four groups. The results suggest that the rats, large animals, and patients have very similar $\alpha / \beta$ ratios. We also noted that the patient data had a large and unbalanced $95 \% \mathrm{CI}$ for all parameters. This is understandable because the data set was unbalanced for the fractionation size. Only 2 data points had a large dose/fraction.

\section{RIM model with fractionation effect from the combined data}

Figure 4 shows the plot of the combined patient and large animal data (group D) with the best fitting of the RIM model. In addition to the 3 original patient data points before correction, animal data from two studies on mice [36] and rats [37] were also presented for comparison. The best fitting parameters and their $95 \% \mathrm{CI}$ were $\alpha / \beta=3.9(3.0,4.9) \mathrm{Gy}$, BED50= $111(101,120) \mathrm{Gy}$, and $k=10.5(6.8,14.2)$. We noted that combining the large animal and patient data did not change the best fitting parameters much, while the $95 \%$ CIs for BED50 and $k$ were remarkably narrowed, which is essential for the applicability of the RIM model. We also noted that the rat and mice data tended to agree with the patient data. However, although the mice or rat data set was from a single experiment, the data were more spread out than the patient and large animal data, possibly due to larger dosimetric uncertainties in small animals. 
Table. 4 Comparison of the fitting parameters for groups A, $\mathrm{B}, \mathrm{C}$ and $\mathrm{D}$

\begin{tabular}{lllll}
\hline Groups & Regression & $\alpha / \beta(\mathrm{Gy})$ & BED50 (Gy) & $k$ \\
\hline A (rats) & Linear regression & 4.1 & 119 & NA \\
& & $(3.2,5.0)$ & $(100,138)$ & \\
& Non-linear regression & 3.6 & 129 & NA \\
& & $(2.6,4.6)$ & $(109,150)$ & \\
B (large animals) & Non-linear regression & 3.9 & 112 & 12 \\
& & $(3.0,4.8)$ & $(101,128)$ & $(7.0,21.0)$ \\
C (patients) & Non-linear regression & 3.7 & 111 & 10.4 \\
& & $(2.2,8.2)$ & $(86.148)$ & $(5.8,19.2)$ \\
D (combined patients and large animals) & Non-linear regression & 3.9 & 111 & 10.5 \\
& & $(3.0,4.9)$ & $(101,120)$ & $(6.8,14.2)$ \\
\hline
\end{tabular}

\section{Discussion}

With the corrections for possible dosimetric and misquotation errors in patient data, and the normalization of the rat data, we have determined that the $\alpha / \beta$ ratios of the spinal cord were 3.6-4.1,3.9, and 3.7 Gy for small animals (rat), large animals, and patients, respectively. We have also determined the three parameters (including their $95 \% \mathrm{CI}$ ) for a logistic RIM model from the combined data. They were $\alpha / \beta=3.9(3.0,4.9) \mathrm{Gy}$, $\operatorname{BED} 50=111(101,120) \mathrm{Gy}$, and $k=10.5(6.8,14.2)$.

The $\alpha / \beta$ ratios are similar between different species, including human beings. These $\alpha / \beta$ ratios of 3.6-4.1 Gy, however, are greater than the previously reported 0.87 Gy [12]. That study had only 7 data points from five patient studies, including 3 data points with a same percentage of RIM of 0 , and 2 data points in dispute (points 1 and 2 in the current study). The statistical power was limited when the data set was fitted to a non-linear equation with three parameters. The data point 1 , which was from the study of breast treatment with 25 Gy in 2 fractions [19], was the most critical point to determine the $\alpha / \beta$ ratio. The change of $\alpha / \beta$ ratio from $0.87 \mathrm{~Gy}$ of that study to $3.7 \mathrm{~Gy}$ of the current study is likely due to the

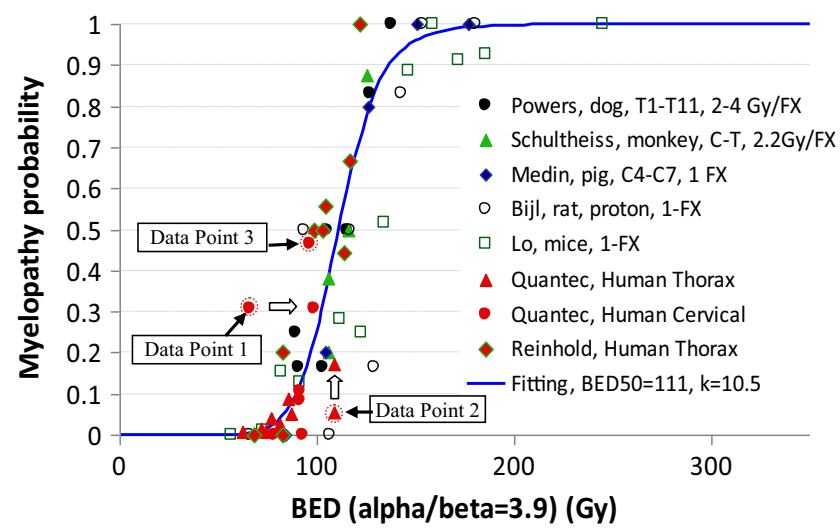

Fig. 4 Fitting the combined (group D) data with a logistic radiationinduced myelopathy (RIM) model. In addition to the 3 patient data points before correction, data from mice and rat studies were also plotted for comparison adjustment of the cord dose from 9.5 to 12 Gyx2. The cord dose of 12 Gyx 2 was estimated based on Monte Carlo simulation in the current study, which is more reliable. The lower bound of the $95 \%$ CI of the $\alpha / \beta$ ratio for the patient group was $2.2 \mathrm{~Gy}$, which is similar to the 2.0 Gy value commonly used clinically for isoeffect calculations.

We noted that the $\alpha / \beta$ ratio was larger, and BED50 smaller, for the $\mathrm{C}$-spine than the $\mathrm{T} / \mathrm{L}$-spine subgroups of the rat studies (Table 3). However, the C-spine subgroup had a shorter follow-up time. The latency of radiation myelopathy was reported to have a bimodal distribution peaking at approximately 9 and 26 months in humans [33]. In rats, the two peaks correspond to two distinct pathologies, with the first peak reflecting white matter injury occurring less than 8 months after radiation exposure, and the second peak reflecting vascular injury occurring between 8 and 18 months after radiation exposure [33]. The latent period depends on the radiation dose and fraction size $[9,36]$. High-dose and single fraction tend to result in shorter latent periods $[9,36]$. Therefore, longer follow-up time would record more events for lower dose at larger fraction numbers $(n)$. As shown in Fig. 2d, this would shift the points of the $\mathrm{C}$-spine subgroup at large $n$ downward, and thus decrease BED50 and increase $\alpha / \beta$ ratios. Therefore, there is a possibility that the larger BED50 and smaller $\alpha / \beta$ ratio observed in the $\mathrm{C}$-spine group was attributed to the shorter follow-up time rather than the anatomic location per se. The non-linear regression also reduced the difference between the two subgroups, suggesting that experimental uncertainty may also contribute to it. The patient data for C- and Tspine was similar, supporting the argument that there is no difference between the $\mathrm{C}$ - and T-spine.

The study showed a BED50 of 111 Gy for the combined and patient data. It corresponds to a D50 of $73.4 \mathrm{~Gy}$ in $2 \mathrm{~Gy} /$ $\mathrm{FX}$, or $19.0 \mathrm{~Gy}$ in single fraction. BED5, which is the BED to induce $5 \%$ of RIM, was calculated to be 83.9 Gy according to Eq. (4) with $k=10.5$. It corresponds to D5 of $55.4 \mathrm{~Gy}$ in $2 \mathrm{~Gy} /$ FX, or 16.2 Gy in single fraction. However, applying these data to clinical SBRT is not straightforward due to two factors. The first factor is the dose volume effect. The cord dose is 
usually not uniform in spinal SRS or SBRT due to the use of intensity modulated techniques. The cord dose is represented by the dose volume histogram and has to be converted into an equivalent uniform dose. The second factor is the setup uncertainty in spinal SRS and SBRT. Even with image guidance, the combined patient setup uncertainty could have a $5 \%$ probability of being greater than $3 \mathrm{~mm}$ due to patient position deformation, uncorrected rotation errors, and patient movement during treatment [38]. Such setup errors may shift the cord into a high-dose region due to the sharp dose falloff between the target and the cord. A RIM model incorporating the setup uncertainty, similar to the approach used in tumor control probability model by Jin et al. [39], may be required to improve the prediction of RIM for spinal SRS or SBRT patients.

\section{Conclusion}

The study suggests that the spinal cord has a similar $\alpha / \beta$ ratio for patients, rats and other large animals, such as dogs, monkeys, and pigs. The $\alpha / \beta$ ratio is close to $3.9 \mathrm{~Gy}$, with a $95 \% \mathrm{CI}$ of $(3.0,4.9)$ for the combined group, and $(2.2,8.2)$ for the patient only group.

Conflict of interest The authors declare that they have no competing interests.

Compliance with ethical standards This article does not contain any studies with human or animal subjects performed by any of the authors

Open Access This article is distributed under the terms of the Creative Commons Attribution 4.0 International License (http:// creativecommons.org/licenses/by/4.0/), which permits unrestricted use, distribution, and reproduction in any medium, provided you give appropriate credit to the original author(s) and the source, provide a link to the Creative Commons license, and indicate if changes were made.

\section{References}

1. Emami B, Lyman J, Brown A et al (1991) Tolerance of normal tissue to therapeutic irradiation. Int J Radiat Oncol Biol Phys 21(1):109-22

2. Kirkpatrick JP, van der Kogel AJ, Schultheiss TE (2010) Radiation dose-volume effects in the spinal cord. Int J Radiat Oncol Biol Phys 76(3 Suppl):S42-9

3. Ryu S, Rock J, Jain R, Lu M, Anderson J, Jin JY, Rosenblum M, Movsas B, Kim JH (2010) Radiosurgical decompression of metastatic epidural compression. Cancer 116(9):2250-7

4. Gerszten PC (2014) Spine metastases: from radiotherapy, surgery, to radiosurgery. Neurosurgery 61(Suppl 1):16-25

5. Ryu S, Pugh SL, Gerszten PC, Yin FF, Timmerman RD, Hitchcock YJ, Movsas B, Kanner AA, Berk LB, Followill DS, Kachnic LA (2014) RTOG 0631 phase $2 / 3$ study of image guided stereotactic radiosurgery for localized (1-3) spine metastases: phase 2 results. Pract Radiat Oncol 4(2):76-81
6. Karger CP, Peschke P, Sanchez-Brandelik R et al (2006) Radiation tolerance of the rat spinal cord after 6 and 18 fractions of photons and carbon ions: experimental results and clinical implications. Int $\mathrm{J}$ Radiat Oncol Biol Phys 66(5):1488-97

7. Ang KK, van der Kogel AJ, van der Schueren E (1983) The effect of small radiation doses on the rat spinal cord: the concept of partial tolerance. Int J Radiat Oncol Biol Phys 9(10):1487-91

8. van der Kogel AJ (1977) Radiation tolerance of the rat spinal cord: time-dose relationships. Radiology 122(2):505-9

9. White A, Hornsey S (1978) Radiation damage to the rat spinal cord: the effect of single and fractionated doses of X rays. Br J Radiol 51(607):515-23

10. Masuda K, Reid BO, Withers HR (1977) Dose effect relationship for epilation and late effects on spinal cord in rats exposed to gamma rays. Radiology 122(1):239-42

11. Leith JT, DeWyngaert JK, Glicksman AS (1981) Radiation myelopathy in the rat: an interpretation of dose effect relationships. Int $\mathrm{J}$ Radiat Oncol Biol Phys 7(12):1673-7

12. Schultheiss TE (2008) The radiation dose-response of the human spinal cord. Int J Radiat Oncol Biol Phys 71(5):1455-9

13. Medin PM, Foster RD, van der Kogel AJ et al (2011) Spinal cord tolerance to single-fraction partial-volume irradiation: a swine model. Int J Radiat Oncol Biol Phys 79(1):226-32

14. Powers BE, Thames HD, Gillette SM et al (1998) Volume effects in the irradiated canine spinal cord: do they exist when the probability of injury is low? Radiother Oncol 46(3):297-306

15. Schultheiss TE, Stephens LC, Ang KK et al (1994) Volume effects in rhesus monkey spinal cord. Int J Radiat Oncol Biol Phys 29(1): $67-72$

16. McCunniff AJ, Lliang MJ (1989) Radiation tolerance of the cervical spinal cord. Int J Radiat Oncol Biol Phys 16:675-678

17. Jeremic BJ, Djuric L, Mijatovic L (1991) Incidence of radiation myelitis of the cervical spinal cord at doses of $5500 \mathrm{cGy}$ or greater. Cancer 68:2138-2141

18. Abbatucci JS, DeLozier T, Quint R et al (1978) Radiation myelopathy of the cervical spinal cord. Time, dose, and volume factors. Int J Radiat Oncol Biol Phys 4:239-248

19. Atkins HL, Tretter P (1966) Time-dose considerations in radiation myelopathy. Acta Radiol Ther Phys Biol 5:79-94

20. Marcus RB Jr, Million RR (1990) The incidence of myelitis after irradiation of the cervical spinal cord. Int J Radiat Oncol Biol Phys 93:3-8

21. Hazra TA, Chandrasekaran MS, Colman M et al (1974) Survival in carcinoma of the lung after a split course of radiotherapy. Br J Radiol 47:464-466

22. Choi NCH, Grillo HC, Gardiello M et al (1980) Basis for new strategies in postoperative radiotherapy of bronchogenic carcinoma. Int J Radiat Oncol Biol Phys 6:31-35

23. Abramson N, Cavanaugh PJ (1973) Short-course radiation therapy in carcinoma of the lung. Radiology 108:685-687

24. Fitzgerald RH, Marks RD, Wallace KM (1982) Chronic radiation myelitis. Radiology 144:609-612

25. Madden FJF, English JSC, Moore AK et al (1979) Split course radiation in inoperable carcinoma of the bronchus. Eur $\mathrm{J}$ Cancer 15:1175-1177

26. Guthrie RT, Ptacek JJ, Hjass AC (1973) Comparative analysis of two regimens of split course radiation in carcinoma of the lung. Am J Roentgenol 117:605-608

27. Dische S, Warburton MF, Sanders MI (1988) Radiation myelitis and survival in the radiotherapy of lung cancer. Int J Radiat Oncol Biol Phys 15:75-81

28. Hatlevoll R, Host H, Kaalhus O (1983) Myelopathy following radiotherapy of bronchial carcinoma with large single fractions: a retrospective study. Int J Radiat Oncol Biol Phys 9:41-44 
29. Eichhorn HJ, Lessel A, Rotte KH (1972) Einfuss verschiedener Bestrahlungsrhythmen auf Tumor-und Normalgewebe in vivo. Strahlentheraphie 146:614-629

30. Scruggs H, El-Mahdi A, Marks RD Jr et al (1974) The results of split-course radiation therapy in cancer of the lung. Am J Roentgenol Radium Ther Nucl Med 121:754-760

31. Macbeth FR, Bolger JJ, Hopwood P et al (1996) Randomized trial of palliative two-fraction versus more intensive 13-fraction radiotherapy for patients with inoperable non-small cell lung cancer and good performance status. Clin Oncol (R Coll Radiol) 8:167-175

32. Drexler G, Veit R, Zankl M (1990) The quality factor for photons. Radiat Prot Dosim 32(2):83-89

33. Medin PM, Boike TP (2011) Spinal cord tolerance in the age of spinal radiosurgery: lessons from preclinical studies. Int J Radiat Oncol Biol Phys 79(5):1302-9

34. Reinhold HS, Kaalen JG, Unger-Gils K (1976) Radiation myelopathy of the thoracic spinal cord. Int J Radiat Oncol Biol Phys 1(7-8):651-7
35. Schultheiss TE, Kun LE, Ang KK et al (1995) Radiation response of the central nervous system. Int J Radiat Oncol Biol Phys 31(5): 1093-112

36. Lo Y, Taylor JM, McBride WH, Withers HR (1993) The effect of fractionated doses of radiation on mouse spinal cord. Int $\mathrm{J}$ Radiat Oncol Biol Phys 27(2):309-17

37. Bijl HP, van Luijk P, Coppes RP et al (2002) Dose-volume effects in the rat cervical spinal cord after proton irradiation. Int $\mathrm{J}$ Radiat Oncol Biol Phys 52(1):205-11

38. Jin JY, Ryu S, Rock J et al (2008) Evaluation of residual patient position variation for spinal radiosurgery using the Novalis image guided system. Med Phys 35(3):1087-93

39. Jin JY, Kong FM, Liu D et al (2011) A TCP model incorporating setup uncertainty and tumor cell density variation in microscopic extension to guide treatment planning. Med Phys 38(1):439-48 